\title{
A New Digital Preoperative Planning Method for Total Hip Arthroplasties
}

\author{
Hendrikus J. A. Crooijmans MSc, Armand M. R. P. Laumen MD, \\ Carola van Pul PhD, Jan B. A. van Mourik MD
}

Received: 27 November 2007 / Accepted: 18 August 2008/Published online: 10 September 2008

(C) The Author(s) 2008. This article is published with open access at Springerlink.com

\begin{abstract}
Preoperative templating is an important part of a THA. The ability to accurately determine magnification of the hip on the radiograph and apply identical magnification to the radiograph and template will improve accuracy of preoperative templating of THA. We designed a templating method using a new way of determining the hip magnification with a linear relationship between magnification of the hip and the reference object on top of the pubis symphysis; the relationship was determined on 50 radiographs. We then compared our method with two other templating methods: an analog method assuming an average hip magnification of $15 \%$ and a digital method
\end{abstract}

Each author certifies that he or she has no commercial associations (eg, consultancies, stock ownership, equity interest, patent/licensing arrangements, etc) that might pose a conflict of interest in connection with the submitted article.

Each author certifies that his or her institution either has waived or does not require approval for the human protocol for this investigation and that all investigations were conducted in conformity with ethical principles of research.

H. J. A. Crooijmans, C. van Pul

Clinical Physics Department, Máxima Medical Center,

Eindhoven/Veldhoven, The Netherlands

H. J. A. Crooijmans

Faculty of Biomedical Engineering, Eindhoven University of Technology, Eindhoven, The Netherlands

H. J. A. Crooijmans ( $\square)$

MR-Physics, Department of Clinical Radiology, University of

Basel, University Hospital, Petersgraben 4, 4031 Basel,

Switzerland

e-mail: H.J.A.Crooijmans@gmail.com

A. M. R. P. Laumen, J. B. A. van Mourik

Department of Orthopaedic Surgery and Traumatology, Máxima

Medical Center, Eindhoven/Veldhoven, The Netherlands determining the hip magnification with a one-to-one relationship between the reference object and the hip. All methods were reproducible. Uniform undersizing occurred when templating with the digital method based on the oneto-one relationship; the analog method best predicted the implanted prosthesis size, closely followed by our new digital templating method; the new method will be particularly applicable for preoperative THA when analog methods are replaced by digital methods.

\section{Introduction}

Preoperative templating forms an important part of THA to determine the size of the prosthesis and position of the center of rotation of the hip before surgery. Accurate preoperative planning improves the procedure's precision [1, $16]$, shortens its duration $[1,22]$, and reduces the incidence of prosthesis loosening [13, 19], and loss of bone stock [13, 14, 19] among other complications (eg, instability, limblength differences, periprosthetic fractures) $[4,7,8,11,13$, $17,21,23,25,30,32]$. The inability to accurately determine magnification of the radiograph is one of the main problems in hard-copy radiograph templating of THA because analog templates with standard magnifications do not allow for compensation of any deviation in the magnification of the hard-copy radiograph. In digital radiograph templating, the template and radiograph can be scaled to obtain identical magnifications. However, that presumes knowledge of the appropriate scaling. The ability to accurately determine the magnification of the hip on the radiograph and to apply identical magnification to the radiograph and template will improve accuracy of preoperative templating of THA. 
One study to assess scaling used an object of known size (reference object) placed adjacent to the hip to determine the hip magnification [12]. When properly placed, the magnification of the reference object represented the magnification of the hip (one-to-one relationship) and thereby enabled accurate preoperative templating. The method required the reference object to be properly placed at the same distance from the detector as the center of rotation of the hip. Alternative methods for correcting for magnification, including using a line as a magnification reference [20], using coins placed at various positions [20, 31 ] as a magnification reference, using software to template digital radiographs $[6,26]$, or using software to template CT data have been described [29]. Limitations of these methods include proper placement of the object (exact similar anteroposterior [AP] position as the hip) and absence of an accurate description of the magnification relationship between the reference object and the hip, which is assumed to be one-to-one. To continue using the same imaging modality (radiography) and obtaining appropriate scaling, this relationship should be determined.

We first established the relationship between the magnification of the reference object on the pubis symphysis and the hip magnification. Next, we devised a new method of templating, using the relationship between magnification of the reference object placed at the pubis symphysis and the center of rotation of the hip to accurately determine the magnification of the hip. We then asked whether our new method would be superior (percentages of exact or \pm 1 size used) to an analog templating method assuming an average magnification of the hip of $15 \%$ and the most frequently used digital method based on a one-to-one relationship for cemented and uncemented prostheses. We also asked whether these three methods would be reproducible, and whether the reproducibility differed between cemented and uncemented prostheses.

\section{Materials and Methods}

Our study consisted of two parts. In the first part of the study, we determined the relationship between magnification of a reference object placed on top of the pubic symphysis and magnification of the hip on AP pelvic radiographs. In the second part we determined which of three methods (one analog hard-copy templating method and two digital templating methods) was the most accurate templating method and if the methods were reproducible. We made these assessments for cemented and uncemented prostheses.

To determine the relationship between magnifications of the hip and the reference object, we initially included 56 patients in a retrospective analysis. We included patients in whom THA was performed by or under the supervision of one of the authors (JBAvM) from December 2005 to August 2006, with a prosthetic head diameter of $28 \mathrm{~mm}$, a postsurgery AP pelvic radiograph with a reference object at the pubis symphysis, and a known prosthesis head size. Six of the 56 patients were excluded because the implanted prosthesis was a Metasul ${ }^{\circledR}$ (Zimmer, Ltd, Swindon, UK) THA. The metal-on-metal construction of this type makes it impossible to obtain adequate measurement of the diameter of the prosthetic head on a radiograph. The patient group then consisted of 50 patients (36 females, 14 males) between 49 and 87 years of age.

Because the reference object and the hip usually are not at the same distance from the detector, scaling one-to-one using a reference object is incorrect unless a correction factor is used. To determine the relationship between magnification of the hip and magnification of the reference object placed on the pubic symphysis, we measured the diameters of the prosthetic head and the reference object on the postsurgery digital AP pelvic radiographs with Agfa Web1000 ${ }^{\mathrm{TM}}$ software (Agfa Healthcare, Mortsel, Belgium). The measured diameters were divided by the object's real diameter $(10 \mathrm{~mm}$ for the reference object, $28 \mathrm{~mm}$ for the prosthetic head) to determine the magnification and the results recorded.

The relationship between magnification of the hip and reference object was estimated with a linear fit performed in SPSS ${ }^{\circledR}$ for Windows ${ }^{\circledR}$ (SPSS Inc, Chicago, IL). Correlation/regression analysis was performed with the linear regression function in the same program. Correlation strength and significance were calculated and determined according to Cohen [5] (small, $0.10<\mathrm{R}<0.29$; medium, $0.30<\mathrm{R}<0.49$; large, $0.49<\mathrm{R}<1.00$ ).

To compare and determine the most accurate templating method, we retrospectively identified 33 patients between 50 and 83 years of age. The study group consisted of 16 uncemented THAs (10 females, six males) and 17 cemented THAs (14 females, three males). We included patients in whom THA was performed by or under the supervision of one of the authors (JBAvM) from December 2005 to October 2006, with a presurgery AP pelvic radiograph with a reference object at the pubic symphysis and a known prosthesis size.

The AP pelvic radiographs were generated with a 100$\mathrm{cm}$ source-detector distance, the patient in the supine position, and focus on the patient's midline, the pelvis, and as much of the femur as possible included. The digital radiograph was stored in the hospital's Picture Archiving and Communications System and printed for use in analog hard-copy templating. The reference object was a massive metal sphere with a diameter of $10 \mathrm{~mm}$ and was positioned at the pubic symphysis of the patient. For a cemented prosthesis, the M.E. Müller ${ }^{\mathrm{TM}}$ Straight Stem (Zimmer, Ltd; 
standard or lateral; five sizes, 7.5-17.5) was used in combination with a Müller ${ }^{\mathrm{TM}}$ Low Profile Cup (Zimmer, Ltd; 13 sizes, 40-64). For an uncemented prosthesis, the CLS ${ }^{\circledR}$ Spotorno ${ }^{\mathrm{TM}}$ Stem (Zimmer, Ltd; CCD angle, $135^{\circ}$ or $145^{\circ}$; 11 sizes, 6-17.5) was used in combination with the Fitmore $^{\mathrm{TM}}$ Shell with stabilization fins with a Fitec polyethylene insert (Zimmer, Ltd; 13 sizes, 40-64). The largest possible prosthesis was chosen within the cortical edges of the femur and the acetabulum.

Analog hard-copy templating was performed using transparent sheets on which the contours of the prosthesis were depicted. The magnification of the templates is $15 \%$ as provided by the prostheses manufacturer (Zimmer, Ltd). The hard-copy radiograph was overlaid with the template and the prosthesis sizes were determined for femoral and acetabular components. All surgeries were preceded by preoperative templating according to the analog hard-copy templating method.

Digital Method 1 was performed with IMPAX ${ }^{\mathrm{TM}}$ ES Orthopaedic Application planning software (Agfa Healthcare). Preliminary to digital templating, we determined magnification of the hip by ascertaining the magnification of the reference object. First, a circle was positioned (integrated in the software) indicating the edges of the reference object. Next, the diameter of the reference object was given and the radiograph was automatically scaled to the magnification of the templates (also integrated in the software). Templating was performed and the implant sizes recorded.

Digital Method 2 was performed identical to Digital Method 1 except for scaling of the radiograph. Instead of using the diameter of the reference object, the diameter of the reference object was measured on the radiograph and used for correction for the difference in object-detector distance between patients by using the linear relationship between magnification of the reference object and the hip (found in Part 1 of the study). The corrected reference object diameter was used as the input when scaling the radiograph to the magnification of the templates. Templating then was performed and the results were recorded.

Templating was performed independently by two orthopaedic surgeons (JBAvM, RPAJ) and two orthopaedic residents (AMRPL, KEdK) to obtain information regarding interobserver variability. Each rater templated all 33 cases with the three methods described previously to obtain information regarding the method accuracy and interobserver variability. Two of the authors (JBAvM, an experienced orthopaedic surgeon, and AMRPL, an orthopaedic resident) templated the 16 uncemented THAs a second time to determine intraobserver variability. All cases were unknown to all raters, and raters were blinded to the results of the previously performed templating and the implanted prosthesis sizes. Interobserver and intraobserver variability were determined by an intraclass correlation (ICC) calculated with the online statistical tool of Mater Research Support Centre [18]. According to this reference, ICC can be interpreted as: 0 to 0.2 indicates poor agreement; 0.3 to 0.4 indicates fair agreement; 0.5 to 0.6 indicates moderate agreement; 0.7 to 0.8 indicates strong agreement; and greater than 0.8 indicates almost perfect agreement.

The literature suggests $a \pm 1$ size estimation of the prosthesis size is adequate for a templating method [1, 3, 6 , $9,10,16,27,30]$. We therefore based our interpretations of appropriate sizes on $\mathrm{a} \pm 1$ size.

The templating results were categorized as either exact planned results or \pm 1 size planned results. Templated prostheses sizes were plotted in a histogram accompanied by the normal distribution curve generated in SPSS ${ }^{\circledR}$ for Windows ${ }^{\circledR}$ (SPSS Inc). Histogram frequency plots with normal distribution curves were generated to illustrate the shape, center, and spread of the distribution of the obtained templating results.

\section{Results}

The relationship between magnification of the reference object $\left(\mathrm{M}_{\text {reference object }}\right)$ and the hip $\left(\mathrm{M}_{\text {hip }}\right)$ differs from a one-to-one relationship (Fig. 1) and is given by

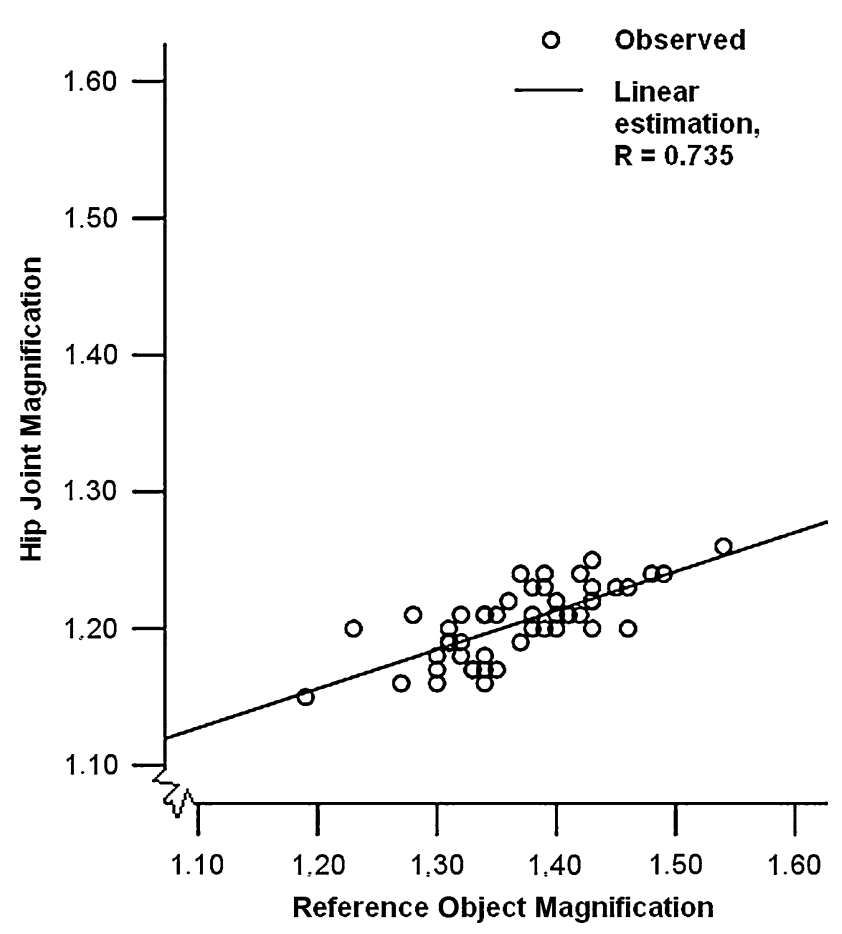

Fig. 1 Hip magnification is correlated with reference object magnification $(R=0.735 ; p<0.001)$ in a data set of 50 relationships. The circles indicate the individual relationships between hip magnification and reference object magnification; the line indicates the correlation between the two. 
$\mathrm{M}_{\text {hip }}=0.8131+0.2857 \mathrm{M}_{\text {reference object. The magnifica- }}$ tions of the reference object correlated $(\mathrm{R}=0.735$; $\mathrm{p}<0.001)$ with those of the hip.

For all prostheses, Digital Method 1 resulted in the lowest percentage of exact planned cases for both components (total). The trend that can be observed is an undersizing when templating with Digital Method 1 and (almost) correct planning when using the analog method or Digital Method 2 (Fig. 2). For the cemented prostheses, the highest percentages of exact planned and of \pm 1 size planned cases were achieved by the analog method for femoral and acetabular components. However, for the acetabular component, we observed a substantial difference between the observers (Table 1). For uncemented

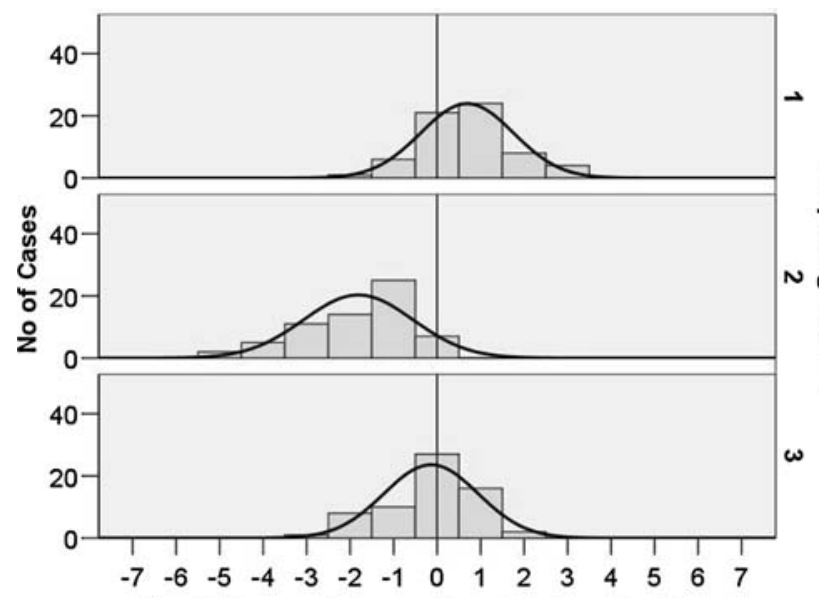

A

Size Difference between Templated and Implanted Uncemented Femoral Prosthesis Component
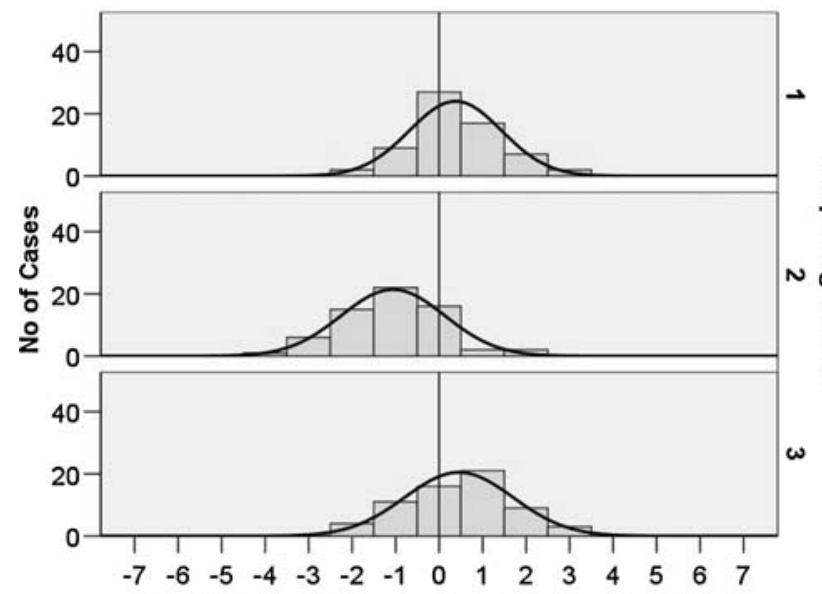

C Size Difference between Templated and Implanted

Fig. 2A-D Histograms and corresponding normal curves of the templating results (Gaussian curves using the mean and standard deviation) show the accuracy of all methods on all types of prostheses components. The data are shown per component: (A) uncemented femoral prosthesis component; (B) cemented femoral prosthesis component; (C) uncemented acetabular prosthesis component; and (D) cemented acetabular prosthesis component. The results of all prostheses, the highest percentages of exact planned cases (total) were obtained with Digital Method 2 when templating femoral components (Table 2). For acetabular components, the highest percentage of exact planned cases (total) was obtained with the analog method.

All methods provided adequate intraobserver agreement (ICC values 0.7 or greater) (Table 3 ). The experienced orthopaedic surgeon and the less experienced resident achieved substantial agreement for intraobserver variability, indicating good reproducible results. ICC values for interobserver variability among the four raters for the analog method and Digital Method 2 were 0.6 or greater; Digital Method 1 was less reliable with ICC values less than 0.6 for interobserver variability (Table 4). The templating results of
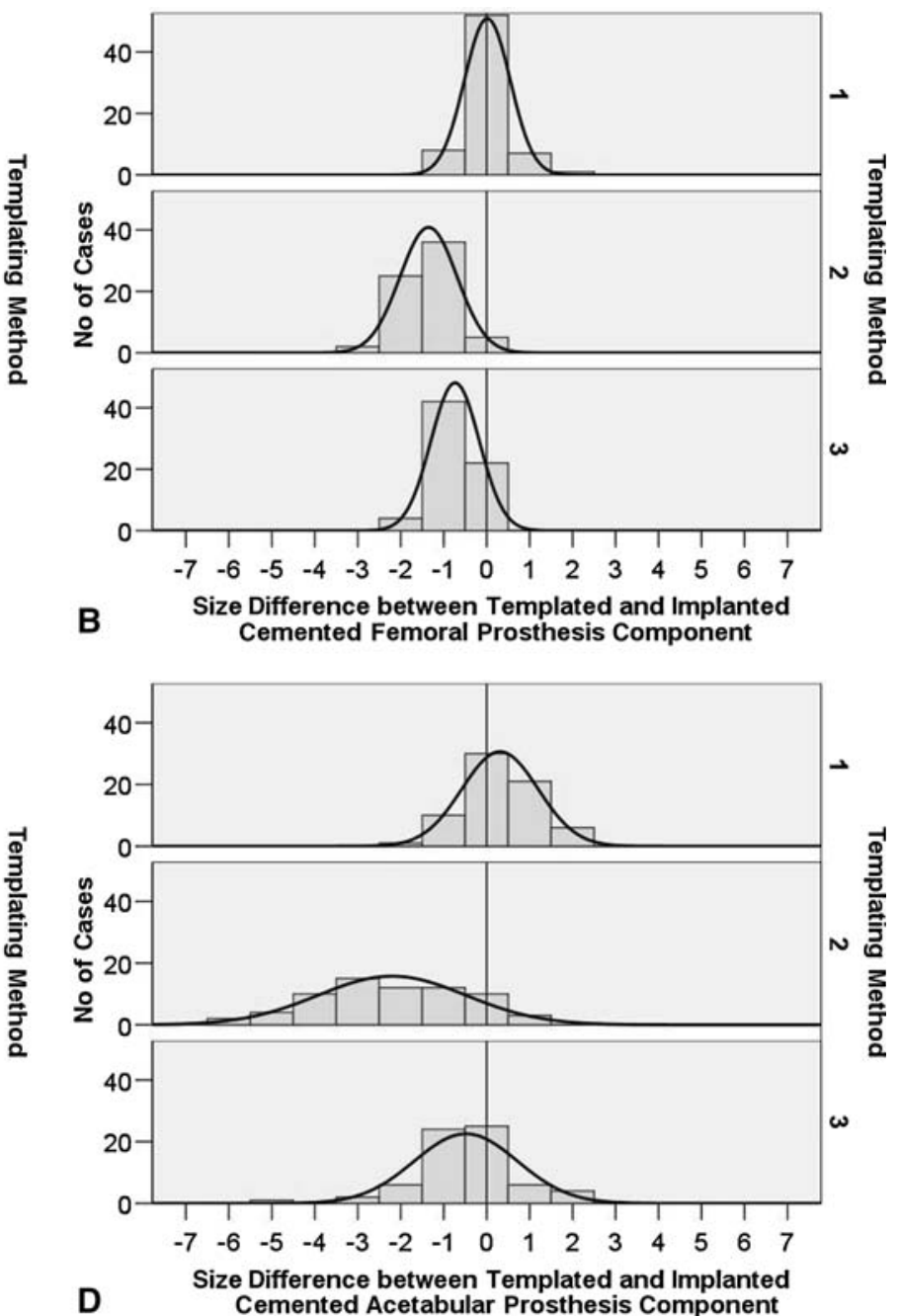

observers (total) are used, and the methods are numbered 1, 2, and 3 for, respectively, the analog method, Digital Method 1, and Digital Method 2. A negative value indicates undersizing of the prosthesis component (the templated prosthesis size is smaller than the implanted prosthesis size); a positive value indicates oversizing of the prosthesis component. 
Table 1. Templating results for cemented prosthesis components

\begin{tabular}{|c|c|c|c|c|c|}
\hline \multirow[t]{2}{*}{ Templating method } & \multirow[t]{2}{*}{ Rater } & \multicolumn{2}{|c|}{ Cemented femoral component } & \multicolumn{2}{|c|}{ Cemented acetabular component } \\
\hline & & $\begin{array}{l}\text { Number of } \\
\text { exact cases }\end{array}$ & $\begin{array}{l}\text { Number of } \pm 1 \\
\text { size cases }\end{array}$ & $\begin{array}{l}\text { Number of } \\
\text { exact cases }\end{array}$ & $\begin{array}{l}\text { Number of } \pm 1 \\
\text { size cases }\end{array}$ \\
\hline & Total number of cases & $17(100 \%)$ & $17(100 \%)$ & $17(100 \%)$ & $17(100 \%)$ \\
\hline \multirow[t]{5}{*}{ Analog } & JBAvM & $15(88.2 \%)$ & $16(94.1 \%)$ & $11(64.7 \%)$ & $17(100 \%)$ \\
\hline & AMRPL & $11(64.7 \%)$ & $17(100 \%)$ & $4(23.5 \%)$ & $10(58.8 \%)$ \\
\hline & RPAJ & $12(70.6 \%)$ & $16(94.1 \%)$ & $6(35.3 \%)$ & $17(100 \%)$ \\
\hline & KEdK & $14(82.4 \%)$ & $17(100 \%)$ & $14(82.4 \%)$ & $17(100 \%)$ \\
\hline & Total $^{\dagger}$ & $52(76.5 \%)$ & $66(97.1 \%)$ & $35(51.5 \%)$ & $61(89.7 \%)$ \\
\hline \multirow[t]{5}{*}{ Digital 1} & JBAvM & $1(5.9 \%)$ & $10(58.8 \%)$ & $1(5.9 \%)$ & $6(35.3 \%)$ \\
\hline & AMRPL & $0(0 \%)$ & $8(47.1 \%)$ & $0(0 \%)$ & $0(0 \%)$ \\
\hline & RPAJ & $3(17.6 \%)$ & $9(52.9 \%)$ & $0(0 \%)$ & $2(11.8 \%)$ \\
\hline & KEdK & $1(5.9 \%)$ & $11(64.7 \%)$ & $9(52.9 \%)$ & $17(100 \%)$ \\
\hline & Total $^{\dagger}$ & $5(7.4 \%)$ & $38(55.9 \%)$ & $10(14.7 \%)$ & $25(36.8 \%)$ \\
\hline \multirow[t]{5}{*}{ Digital 2} & JBAvM & $8(47.1 \%)$ & $17(100 \%)$ & $7(41.2 \%)$ & $14(82.4 \%)$ \\
\hline & AMRPL & $1(5.9 \%)$ & $14(82.4 \%)$ & $7(41.2 \%)$ & $15(88.2 \%)$ \\
\hline & RPAJ & $8(47.1 \%)$ & $17(100 \%)$ & $4(23.5 \%)$ & $12(70.6 \%)$ \\
\hline & KEdK & $5(29.4 \%)$ & $16(94.1 \%)$ & $7(41.2 \%)$ & $14(82.4 \%)$ \\
\hline & Total $^{\dagger}$ & $22(32.4 \%)$ & $64(94.1 \%)$ & $25(36.8 \%)$ & $55(80.9 \%)$ \\
\hline
\end{tabular}

${ }^{*} \pm 1$ size includes the exact templated cases; ${ }^{\dagger}$ total of all raters.

Table 2. Templating results for uncemented prosthesis components

\begin{tabular}{|c|c|c|c|c|c|}
\hline \multirow[t]{2}{*}{ Templating method } & \multirow[t]{2}{*}{ Rater } & \multicolumn{2}{|c|}{ Uncemented femoral component } & \multicolumn{2}{|c|}{ Uncemented acetabular component } \\
\hline & & $\begin{array}{l}\text { Number of } \\
\text { exact cases }\end{array}$ & $\begin{array}{l}\text { Number of } \pm 1 \\
\text { size cases }\end{array}$ & $\begin{array}{l}\text { Number of } \\
\text { exact cases }\end{array}$ & $\begin{array}{l}\text { Number of } \pm 1 \\
\text { size cases }\end{array}$ \\
\hline \multirow{6}{*}{ Analog } & Total number of cases & $16(100 \%)$ & $16(100 \%)$ & $16(100 \%)$ & $16(100 \%)$ \\
\hline & JBAvM & $7(43.8 \%)$ & $15(93.8 \%)$ & $6(37.5 \%)$ & $14(87.5 \%)$ \\
\hline & AMRPL & $8(50.0 \%)$ & $15(93.8 \%)$ & $4(25.0 \%)$ & $10(62.5 \%)$ \\
\hline & RPAJ & $3(18.8 \%)$ & $14(87.5 \%)$ & $8(50.0 \%)$ & $15(93.8 \%)$ \\
\hline & KEdK & $3(18.8 \%)$ & $10(62.5 \%)$ & $9(56.3 \%)$ & $14(87.5 \%)$ \\
\hline & $\operatorname{Total}^{\dagger}$ & $21(32.8 \%)$ & $54(84.4 \%)$ & $27(42.2 \%)$ & $53(82.8 \%)$ \\
\hline \multirow[t]{5}{*}{ Digital 1} & JBAvM & $2(12.5 \%)$ & $10(62.5 \%)$ & $6(37.5 \%)$ & $12(75.0 \%)$ \\
\hline & AMRPL & $0(0.0 \%)$ & $1(6.3 \%)$ & $1(6.3 \%)$ & $5(31.3 \%)$ \\
\hline & RPAJ & $4(25.0 \%)$ & $13(81.3 \%)$ & $2(12.5 \%)$ & $11(68.8 \%)$ \\
\hline & KEdK & $1(6.3 \%)$ & $8(50.0 \%)$ & $7(43.8 \%)$ & $12(75.0 \%)$ \\
\hline & $\operatorname{Total}^{\dagger}$ & $7(10.9 \%)$ & $32(50.0 \%)$ & $16(25.0 \%)$ & $40(62.5 \%)$ \\
\hline \multirow[t]{5}{*}{ Digital 2} & JBAvM & $8(50.0 \%)$ & $13(81.3 \%)$ & $4(25.0 \%)$ & $14(87.5 \%)$ \\
\hline & AMRPL & $5(31.3 \%)$ & $11(68.8 \%)$ & $5(31.3 \%)$ & $12(75.0 \%)$ \\
\hline & RPAJ & $7(43.8 \%)$ & $15(93.8 \%)$ & $4(25.0 \%)$ & $13(81.3 \%)$ \\
\hline & KEdK & $7(43.8 \%)$ & $14(87.5 \%)$ & $3(18.8 \%)$ & $9(56.3 \%)$ \\
\hline & Total $^{\dagger}$ & $27(42.2 \%)$ & $53(82.8 \%)$ & $16(25.0 \%)$ & $48(75.0 \%)$ \\
\hline
\end{tabular}

${ }^{*} \pm 1$ size includes the exact templated cases; ${ }^{\dagger}$ total of all raters.

the greater and lesser experienced observers for Digital Method 1 and Digital Method 2 had ICC values greater than 0.6 (Table 5), whereas the analog method had lower reproducibility with values between 0.52 and 0.83 for the ICC. For Digital Method 1, ICC values for interobserver variability between two of the observers (Table 5) were 
Table 3. ICC values for uncemented prosthesis intraobserver variability

\begin{tabular}{lllll}
\hline Rater & $\begin{array}{l}\text { Prosthesis } \\
\text { component }\end{array}$ & \multicolumn{2}{l}{ Method } & \\
\cline { 3 - 5 } & & Analog & Digital 1 & Digital 2 \\
\hline JBAvM & Femoral & 0.93 & 0.81 & 0.92 \\
& Acetabular & 0.82 & 0.94 & 0.91 \\
AMRPL & Femoral & 0.85 & 0.85 & 0.70 \\
& Acetabular & 0.77 & 0.90 & 0.74 \\
\hline
\end{tabular}

ICC $=$ intraclass correlation coefficient.

Table 4. ICC values for interobserver variability of all four raters

\begin{tabular}{|c|c|c|c|c|}
\hline \multirow[t]{2}{*}{ Prosthesis type } & \multirow{2}{*}{$\begin{array}{l}\text { Prosthesis } \\
\text { component }\end{array}$} & \multicolumn{3}{|l|}{ Method } \\
\hline & & Analog & Digital 1 & Digital 2 \\
\hline \multirow[t]{2}{*}{ Cemented } & Femoral & 0.84 & 0.58 & 0.72 \\
\hline & Acetabular & 0.60 & 0.52 & 0.68 \\
\hline \multirow[t]{2}{*}{ Uncemented } & Femoral & 0.77 & 0.59 & 0.61 \\
\hline & Acetabular & 0.72 & 0.84 & 0.84 \\
\hline
\end{tabular}

ICC $=$ intraclass correlation coefficient.

Table 5. ICC values for interobserver variability of raters JBAvM and RPAJ

\begin{tabular}{|c|c|c|c|c|}
\hline \multirow[t]{2}{*}{ Prosthesis type } & \multirow{2}{*}{$\begin{array}{l}\text { Prosthesis } \\
\text { component }\end{array}$} & \multicolumn{3}{|l|}{ Method } \\
\hline & & Analog & Digital 1 & Digital 2 \\
\hline \multirow[t]{2}{*}{ Cemented } & Femoral & 0.83 & 1.00 & 0.71 \\
\hline & Acetabular & 0.52 & 0.64 & 0.76 \\
\hline \multirow[t]{2}{*}{ Uncemented } & Femoral & 0.65 & 0.74 & 0.70 \\
\hline & Acetabular & 0.60 & 0.89 & 0.80 \\
\hline
\end{tabular}

ICC $=$ intraclass correlation coefficient.

larger than among all four raters (Table 4), indicating larger agreement between these two observers than among all four.

\section{Discussion}

Incorrect preoperative templating of a THA might lead to inappropriate implant size and position $[1,4,7,8,11,13$, $14,16,17,19,21-23,25,30,32]$, and revision of the prosthesis might be needed. Preoperative analog $[3,4,7,9$ $11,16,25,27,29]$, and digital $[2,6,9,15,20,22,24,27-$ 30] templating methods have been studied. Unfortunately, none of these digital templating methods has become a standard method used in clinical application. Given the importance of correct preoperative templating and the possibility of correction with digital radiography, we developed an improved two-dimensional templating method. In this study, we tried to answer the following questions: (1) What is the relationship between magnification of the reference object on the pubis symphysis and hip magnification?; (2) How does our method compare with other investigated methods according to accuracy?; (3) Are these methods reproducible?; and (4) Are results different for cemented and uncemented prostheses?

There are some limitations to our study. First, we assumed the implanted prosthesis was always the optimal size, which might not have been the case. However, all methods were compared with these prostheses sizes, and the influence of any suboptimal size therefore would be similar for each method, making it possible to compare the outcomes of the studies with one another. Second, all surgeries were preceded by preoperative analog templating. This might lead to higher correspondence of the analog method outcome with the implanted prosthesis size in our study than when preoperative templating was performed according to any other method or not at all. We believe preoperative planning is important, and we have used templating for many years. Therefore, the patients included in this study had surgery based on the outcome of preoperative planning according to the analog method. This might bias the implanted prosthesis size used as the reference value in this study. This bias might result in a larger number of cases showing agreement between templated and implanted prosthesis size with the analog method. We considered this possible bias when coming to our conclusions. Third, familiarity with the analog method might lead to higher reproducibility of the results with the analog method. The fourth limitation is incomplete blinding of one of the authors (JBAvM) to all patients. However, given the substantial agreement between two of the authors (JBAvM, RPAJ), this would be a small influence. Our relatively small sample size is a fifth limitation to the study; larger study groups might make the outcomes more reliable. Finally, although we do not see this as a limitation peculiar to our study, no templating method based on AP radiographs can directly determine the absolute magnification (and thus the absolute size of the acetabular component), although we can correct for a difference in magnification caused by a difference in AP positioning between the reference object and the hip. Templating of the AP position of a prosthesis is an additional possibility when templating with Digital Method 2 compared with other templating methods based on AP radiographs.

We found a linear relationship between magnification of the reference object at the pubis symphysis and the hip. The use of this relationship provides an adequate estimate of hip magnification based on the reference object magnification and therefore was used in the design of Digital Method 2. This is a new method of magnification determination deflecting the one-to-one relation used in other studies $[6,12,20,26,31]$. 
Table 6. Published templating results

\begin{tabular}{|c|c|c|c|c|c|}
\hline \multirow[t]{2}{*}{ Templating method } & \multirow[t]{2}{*}{ Study } & \multicolumn{4}{|c|}{ Percentage of templated cases $(+1$ size range* $)$} \\
\hline & & $\begin{array}{l}\text { Femoral } \\
\text { cemented }\end{array}$ & $\begin{array}{l}\text { Acetabular } \\
\text { cemented }\end{array}$ & $\begin{array}{l}\text { Femoral } \\
\text { cementless }\end{array}$ & $\begin{array}{l}\text { Acetabular } \\
\text { cementless }\end{array}$ \\
\hline \multirow[t]{6}{*}{ Analog } & Knight and Atwater [16] & $100.0 \%$ & & $85.0 \%$ & $96.0 \%$ \\
\hline & Viceconti et al. $[28]^{\dagger}$ & $82.8 \%$ & $69.0 \%$ & $82.8 \%$ & $69.0 \%$ \\
\hline & Goldstein et al. $[8]^{\dagger}$ & $99.2 \%$ & $99.2 \%$ & $99.2 \%$ & $99.2 \%$ \\
\hline & The et al. [27] & $89.0 \%$ & $73.0 \%$ & $52.0 \%$ & $64.0 \%$ \\
\hline & Carter et al. [3] & & & $88.3 \%$ & \\
\hline & Eggli et al. [7] & $94.0 \%$ & & & $81.0 \%$ \\
\hline \multirow[t]{3}{*}{ Digital two-dimensional } & The et al. [27] & $79.0 \%$ & $72.0 \%$ & $66.0 \%$ & $52.0 \%$ \\
\hline & Davila et al. [6] ${ }^{\dagger}$ & $72.0 \%$ & $86.0 \%$ & $72.0 \%$ & $86.0 \%$ \\
\hline & Goldstein et al. [8] & $98.0 \%$ & & & $97.0 \%$ \\
\hline Digital three-dimensional & Viceconti et al. $[28]^{\dagger}$ & $86.2 \%$ & $93.1 \%$ & $86.2 \%$ & $93.1 \%$ \\
\hline
\end{tabular}

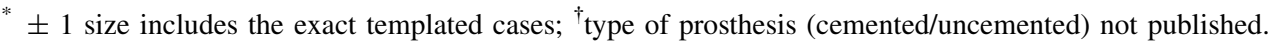

The analog method was the best according to the percentages of cases planned within the range of \pm 1 size, closely followed by Digital Method 2, with a maximal difference of $8.8 \%$ of the cases. Digital Method 1 planned sizes appeared within the \pm 1 size range less often than sizes planned with the analog method for the same cases. The difference was at least $20.3 \%$ of the total amount of templated cases (Tables 1,2), and therefore the least successful method was Digital Method 1. Our analog method data (Tables 1,2) were comparable to those of other published studies (Table 6). Our Digital Method 2 (Tables 1,2) was better in templating femoral components than other published digital templating methods using digital radiographs and similar in templating acetabular components (Table 6). However, direct comparison of the different studies is difficult, because the possible steps in the component sizes differ for different types of prostheses; however, comparison within one study should be reliable.

We found intraobserver variability ICC values similar to those for other analog and digital planning methods [20]. The ICC values for interobserver variability of Digital Method 1 and Digital Method 2 were higher than other published values [20]. ICC values of Digital Method 1 were lower than those of Digital Method 2, which might be attributable to the undersizing obtained with Digital Method 1. The determined prosthesis sizes are, on average, much smaller than usual, making the orthopaedic surgeon doubt the determined result and automatically, because of experience with the average prosthesis size, in some cases choose a larger size. All methods had at least moderate [18] reproducibility (ICC values greater than 0.5 ) for interobserver and intraobserver variability. For the cemented and the uncemented cases, the analog method was best, closely followed by our Digital Method 2. The percentages of cases planned in the \pm 1 size range generally were higher for cemented than for uncemented prostheses because of the smaller step size between sizes for uncemented prostheses.

Implantations of the prostheses were based on the analog method, and as stated before, this may have biased the results in favor of the analog method for accuracy, which could explain the difference in the amount of correctly planned cases between the analog method and Digital Method 2. Therefore, we believe the difference obtained between the analog method and Digital Method 2 is small and Digital Method 2 is almost as accurate as the analog method, suggesting our method could be the digital templating method used when analog templating is replaced by digital templating.

Acknowledgments We thank Karin E. de Kroon, MD, and Rob P. A. Janssen, MD, for the effort and time to perform templating on all subjects with all methods, and Janno Kuipers, BSc, for help and support in acquiring all hard-copy radiographs. We also thank Pieter F. F. Wijn, PhD, for supervision and support in performing this study.

Open Access This article is distributed under the terms of the Creative Commons Attribution Noncommercial License which permits any noncommercial use, distribution, and reproduction in any medium, provided the original author(s) and source are credited.

\section{References}

1. Blackley HR, Howell GE, Rorabeck CH. Planning and management of the difficult primary hip replacement: preoperative planning and technical considerations. Instr Course Lect. 2000;49:3-11.

2. Bono JV. Digital templating in total hip arthroplasty. J Bone Joint Surg Am. 2004;86:118-122.

3. Carter LW, Stovall DO, Young TR. Determination of accuracy of preoperative templating of noncemented femoral prosthesis. J Arthroplasty. 1995;10:507-513.

4. Cech O, Fassbender M, Kirschner P, Rozkydal Z. [Preoperative planning and surgical technic in achieving stability and leg length 
equality in total hip joint arthroplasty] (in Czech). Acta Chir Orthop Traumatol Cech. 2002;69:362-368.

5. Cohen J. Statistical Power Analysis for the Behavioral Sciences. 2nd ed. Hillsdale, NJ: Lawrence Erlbaum Associates, Inc Publishers; 1988.

6. Davila JA, Kransdorf MJ, Duffy GP. Surgical planning of total hip arthroplasty: accuracy of computer-assisted EndoMap software in predicting component size. Skeletal Radiol. 2006;35:390-393.

7. Eggli S, Pisan M, Muller ME. The value of preoperative planning for total hip arthroplasty. J Bone Joint Surg Br. 1998;80:382-390.

8. Goldstein WM, Gordon A, Branson JJ. Leg length inequality in total hip arthroplasty. Orthopedics. 2005;28(9 suppl):s1037-s1040.

9. González Della Valle A, Comba F, Taveras N, Salvati EA. The utility and precision of analogue and digital preoperative planning for total hip arthroplasty. Int Orthop. 2008;32:289-294.

10. González Della Valle A, Slullitel G, Piccaluga F, Salvati EA. The precision and usefulness of preoperative planning for cemented and hybrid primary total hip arthroplasty. J Arthroplasty. 2005;20:51-58.

11. Goodman SB, Huene DS, Imrie S. Preoperative templating for the equalization of leg length in total hip arthroplasty. Contemp Orthop. 1992;24:703-710.

12. Gorski JM, Schwartz L. A device to measure x-ray magnification in preoperative planning for cementless arthroplasty. Clin Orthop Relat Res. 1986;202:302-306.

13. Haddad FS, Masri BA, Garbuz DS, Duncan CP. The prevention of periprosthetic fractures in total hip and knee arthroplasty. Orthop Clin North Am. 1999;30:191-207.

14. Haddad FS, Masri BA, Garbuz DS, Duncan CP. Femoral bone loss in total hip arthroplasty: classification and preoperative planning. Instr Course Lect. 2000;49:83-96.

15. Hananouchi $T$, Sugano N, Nakamura N, Nishii T, Miki $H$, Yamamura M, Yoshikawa H. Preoperative templating of femoral components on plain X-rays: Rotational evaluation with synthetic X-rays on ORTHODOC. Arch Orthop Trauma Surg. 2007;127:381-385.

16. Knight JL, Atwater RD. Preoperative planning for total hip arthroplasty: quantitating its utility and precision. $J$ Arthroplasty. 1992;7:403-409.

17. Lindgren JU, Rysavy J. Restoration of femoral offset during hip replacement: a radiographic cadaver study. Acta Orthop Scand. 1992;63:407-410.

18. Mater Research Support Centre. Statistical Tools. Available at: www.materresearch.org/mrsc/Resources/StatTools/. Accessed April 2007.
19. Müller ME. Lessons of 30 years of total hip arthroplasty. Clin Orthop Relat Res. 1992;274:12-21.

20. Oddy M, Jones M, Pendegrass C, Pilling J, Wimhurst J. Assessment of reproducibility and accuracy in templating hybrid total hip arthroplasty using digital radiographs. J Bone Joint Surg Br. 2006;88:581-585.

21. Rubash HE, Parvataneni HK. The pants too short, the leg too long: leg length inequality after THA. Orthopedics. 2007;30:764765 .

22. Schiffers N, Schkommodau E, Portheine F, Radermacher K, Staudte HW. [Planning and performance of orthopedic surgery with the help of individual templates] (in German). Orthopäde. 2000;29:636-640.

23. Schmalzried TP. Preoperative templating and biomechanics in total hip arthroplasty. Orthopedics. 2005;28(8 suppl):s849-s851.

24. Sugano N, Ohzono K, Nishii T, Haraguchi K, Sakai T, Ochi T. Computed-tomography-based computer preoperative planning for total hip arthroplasty. Comput Aided Surg. 1998;3:320-324.

25. Suh KT, Cheon SJ, Kim DW. Comparison of preoperative templating with postoperative assessment in cementless total hip arthroplasty. Acta Orthop Scand. 2004;75:40-44.

26. The B, Diercks R, Stewart R, van Ooijen P, van Horn J. Digital correction of magnification in pelvic $\mathrm{X}$-rays for preoperative planning of hip joint replacements: theoretical development and clinical results of a new protocol. Med Phys. 2005;32:2580-2589.

27. The B, Diercks R, van Ooijen P, Van Horn JR. Comparison of analog and digital preoperative planning in total hip and knee arthroplasty. Acta Orthop. 2005;76:78-84.

28. Viceconti M, Chiarini A, Testi D, Taddei F, Bordini B, Traina F, Toni A. New aspects and approaches in pre-operative planning of hip reconstruction: a computer simulation. Langenbecks Arch Surg. 2004;389:400-404.

29. Viceconti M, Lattanzi R, Antonietti B, Paderni S, Olmi R, Sudanese A, Toni A. CT-based surgical planning software improves the accuracy of total hip replacement preoperative planning. Med Eng Phys. 2003;25:371-377.

30. Wedemeyer C, Quitmann H, Xu J, Heep H, von Knoch M, Saxler G. Digital templating in total hip arthroplasty with the Mayo stem. Arch Orthop Trauma Surg. 2007 Nov 10 [Epub ahead of print].

31. Wimsey S, Pickard R, Shaw G. Accurate scaling of digital radiographs of the pelvis: a prospective trial of two methods. J Bone Joint Surg Br. 2006;88:1508-1512.

32. Woolson ST. Leg length equalization during total hip replacement. Orthopedics. 1990;13:17-21. 